\title{
2 Survey on studies about model uncertainties in small body explorations
}

\author{
Jinglang Fenga ${ }^{a^{*}}$, Xiyun Hou ${ }^{\mathrm{a}}$ and Roberto Armellin ${ }^{\mathrm{b}}$ \\ a School of Astronomy and Space Science, Nanjing University, China \\ ${ }^{\text {b }}$ Space Research Center, Surrey University, United Kindom \\ *fjlangabc@gmail.com
}

\section{Abstract}

Currently, the explorations of small solar system bodies (asteroids and comets) have become more and more popular. Due to the limited measurement capability and irregular shape and diverse spin status of the small body, uncertainties on the parameters of the system and s/c executions are a practical and troublesome problem for mission design and operations. The sample-based Monte Carlo simulation is primarily used to propagate and analyze the effects of these uncertainties on the surrounding orbital motion. However, it is generally time-consuming because of large samples required by the highly nonlinear dynamics. New methods need to be applied for balancing computational efficiency and accuracy. To motivate this research area and facilitate the mission design process, this review firstly discusses the dynamical models and the different methods of modeling the mostly related gravitational and non-gravitational forces. Then the main uncertainties in these force models are classified and analyzed, including approaching, orbiting and landing. Then the linear and nonlinear uncertainty propagation methods are described, together with their advantages and drawbacks. Typical mission examples and the associated uncertainty analysis, in terms of methods and outcomes, are summarized. Future research efforts are emphasized in terms of complete modelling, new mission scenarios, and application of (semi-) analytical methods in small body explorations.

Keywords: small body explorations; uncertainty analysis; Monte Carlo simulation; semi-analytical methods; micro-probe

Acronym list 
1 CNSA: China National Space Administration

2 DA: Differential Algebra

3 ESA: European Space Agency

4 FPE: Fokker-Plank differential Equation

5 GNC: Guidance, Navigation and Control

6 JAXA: Japan Aerospace Exploration Agency

7 LAM: Laboratoire d'Astrophysique de Marseille

8 MC: Monte Carlo

9 NASA: National Aeronautics and Space Administration

10 OD: Orbit Determination

11 ODE: Ordinary Differential Equation

12 PC: Polynomial Chaos

13 PDF: Probability Density Function

14 SRP: Solar Radiation Pressure

15 STM: State Transition Matrix

16 STO: Solar Terminator Orbit

17 STT: State Transition Tensor

18 TAG: Touch and Go

19 UT: Unscented Transformation

20

\section{Introduction}

22 Missions to small solar system bodies (asteroids and comets) have got much attention

23 in recent years. For the design of such missions, an important issue is to identify the

24 dynamical environment for a spacecraft's (s/c) rendezvous, approaching, orbiting 25 around and even landing on the body, which usually possesses an irregular gravity 26 field. This gives rise to a great challenge for mission design, not only with regard to

27 the highly nonlinear orbital dynamics that has been extensively addressed [1], but 28 also with regard to the capability of accurately determining the physical parameters 29 of the body, for example, mass, shape, gravity, rotation, density, etc.

30 In the early days, the small bodies could only be detected and measured by ground- 
1 based (i.e., optical and radar) observations. The body's overall size, shape, spin period,

2 brightness and orbit could be estimated albeit with limited accuracies. Since the

3 1990s, distant flybys were practiced by various space agencies resulting in significant

4 improvement in the estimation of asteroids mass and density. For example the Galileo

5 (NASA) flyby of 243 Ida in 1993 [2], Rosetta (ESA) flyby of 2867 Šteins [3], and the

6 Chang'e (CNSA) flyby of 4179 Toutatis in 2012 [4]. Later on, rendezvous missions, e.g.

7 NASA's NEAR and Dawn and JAXA's Hayabusa, explored asteroids in detail at close

8 distance, greatly advancing our understanding of these small bodies. While orbiting

9 the asteroid 433 Eros in 2000, NEAR determined its gravity, mass, spin rate and

10 orientation, density and internal mass distribution [5]. In 2005, Hayabusa

11 characterized 25143 Itokawa's surface thoroughly and provided the observations to

12 develop a precise model of its shape [6]. Moreover, it touched down on the asteroid,

13 collected samples and returned them to Earth for the first time [7].

14 Nevertheless, before close encounter or arrival, the mass of small bodies cannot be

15 reliably estimated. The nucleus of comets is usually surrounded by the injected 16 dusts/gas (i.e. coma), whose densities and speeds cannot be accurately measured.

17 Thus, large uncertainties exist in the s/c flight environment, especially during its 18 rendezvous and approaching phases. Therefore, an insufficient quantification of 19 these uncertainties could lead to erroneous trade-off analyses and ultimately 20 inappropriate design of mission strategies. In the mission's preliminary design 21 process, the focus should be put on characterizing and minimizing the impact of 22 uncertainties on the design and on the performance of space systems [8].

23 There are already extensive studies about uncertainty and sensitivity analysis in 24 many aspects of science and engineering. For their detailed characterizations, the 25 reader can refer to $[9,10,11]$. Specifically, there is one review about the particular 26 topic on different uncertainty propagation methods, and their applications in space 27 situation awareness (SSA) and space flight mission designs [12], primarily focusing 28 on orbital motion around Earth. Different from these studies about Earth missions, 29 this survey provides an overview on the specific topic of the uncertainty problems of $30 \mathrm{~s} / \mathrm{c}$ 's motion around small solar system bodies for complementation. The outline of 
1 this survey is organized as follows. Section 2 describes the dynamics basis, the mostly 2 related forces and their various modeling methods. Section 3 introduces the 3 classification of uncertainties of these forces in small body explorations. Section 4

4 presents an overview on the current status of typical small body missions and the

5 related uncertainty investigations. Section 5 indicates future research prospects and

6 challenges in both new mission scenarios and new methods. Section 6 concludes this

7 survey.

\section{The dynamics}

92.1 The equation of motion

10 For the specific topic of small body explorations, the corresponding dynamical 11 system can be described as

$$
\ddot{\boldsymbol{x}}(t)=\boldsymbol{F}(\boldsymbol{x}, t)=\boldsymbol{F}_{\text {deter }}(\boldsymbol{x}, t)+\boldsymbol{F}_{\text {non-deter }}(t)
$$

13 where

$$
\boldsymbol{F}(\boldsymbol{x}, t)=\boldsymbol{f}_{\text {central }}+\boldsymbol{f}_{\text {non-spherical }}+\boldsymbol{f}_{\text {srp }}+\boldsymbol{f}_{\text {outgassing }}+\boldsymbol{f}_{\text {thruster } \cdots}
$$

15 includes all the possible forces that act on the $\mathrm{s} / \mathrm{c}$ and the modeling of the primary

16 forces is introduced in later sections. The deterministic and non-deterministic parts 17 are represented by $\boldsymbol{F}_{\text {deter }}(\boldsymbol{x}, t)$ and $\boldsymbol{F}_{\text {non-deter }}(t)$, respectively. For motion around 18 the Earth, the solar radiation pressure (SRP) and the atmospheric drag are the main 19 sources for model uncertainties. However, for motion around small bodies, both $20 \boldsymbol{f}_{\text {central }}$ and $\boldsymbol{f}_{\text {non-spherical }}$ include large uncertainties, especially for the non21 spherical part.

\subsection{The rotation status of the small body}

23 The rotation rate of asteroids is related to the size of the body and can range from 24 extremely fast rotation rates $\left(2000 \mathrm{DO}_{8}\right.$ with rotation period of $1.3 \mathrm{~min}$ ) to extremely 25 slow ones (288 Glauke with rotation period of 1200 hours) [13]. Slowly spinning 26 bodies in most cases are large rubble-pile ones because otherwise they might break

27 up if they rotate too fast. In contrast, a fastly rotating body is usually a small asteroid 28 primarily made of monolithic rocks. Currently, some small asteroids, such as Itokawa, 29 Ryugu and Bennu, are also demonstrated to be rubble-pile bodies by rendezvous 30 missions that will be introduced in later sections. A small fraction of asteroids exhibit 
non-principal-axis rotation in which the rotation axis shows an additional precession

2 and nutation, usually referred to as 'tumbling' motion [14, 15, 16]. Toutatis is one

3 example that was observed by Ostro [17] with such kind of rotation, and was

4 confirmed by the flyby data of Chang'e-2 [18]. The rotation status of a comet shares

5 many similarities with that of the asteroid. However, one major distinction is that

6 there is a torque from outgassing, especially during its perihelion passage. The

7 rotational state typically evolves slowly in time and this is highly dependent on the

8 structure and direction of the outgassing and its distribution on the surface of the

9 comet. Detailed study on this topic can be found in [19].

10 The rotational status of a small body has a great influence on the orbital motion,

11 which is more complicated than that of planets and natural moons (larger and

12 spherical). For instance, a fast rotation, on one side, tends to average out the

13 perturbations from the irregular gravity. Moreover, a tumbling motion makes the

14 dynamics time varying and the orbit design difficult, the detailed discussions of which

15 are addressed in [1].

$16 \quad 2.3$ Modeling of gravity field

17 The irregular shape of a small body induces a complex gravitational field, which is 18 responsible for a strongly perturbed dynamical environment compared with that of 19 planets. As a result of such irregularities, the design and maintenance of suitable s/c 20 orbits can be very challenging, as the s/c might escape from or impact on these bodies 21 due to the perturbations. In addition, with the increase of orbital radius, the s/c's 22 motion is less affected by the perturbation of the irregular gravity. The irregular 23 gravity field can typically be represented by three different methods.

24 (a) Spherical harmonics expansion

25 The non-spherical gravitational potential can be expressed as an infinite spherical 26 harmonics series expansion. Therefore, the Laplace equation of the gravity potential 27 can be solved in terms of spherical coordinates, and it was written as [20]

$$
V=\frac{G M}{r}\left\{1+\sum_{n \geq 1}^{\infty} \sum_{m=0}^{n}\left(\frac{R e}{r}\right)^{n} P_{n m}(\sin (\theta))\left[C_{n m} \cos (m \lambda)+S_{n m} \sin (m \lambda)\right]\right\}
$$

29 where the reference radius $R_{e}$ (usually chosen as the mean radius for a spherical 
1 body) is here chosen as the maximum radius of the irregular and elongated body; ( $\lambda$,

$2 \theta$ ) are the longitude and latitude, respectively; and $r$ is the distance from the $\mathrm{s} / \mathrm{c}$ or

3 the particle to the center of mass of the body. $P_{n m}$ is the associated Legendre

4 functions. $C_{n m}$ and $S_{n m}$ are the spherical harmonic coefficients that are determined

5 by the mass distribution within the body. These coefficients can be expressed in terms

6 of inertia integrals [21], and they can be estimated, with some level of accuracy, from

7 radar/optical observations. Since this method actually expands the potential into a

8 spherical harmonics series, it diverges within the circumscribing sphere ${ }^{1}$ and,

9 therefore, the potential $V$ is only valid outside this sphere.

10 (b) Polyhedron model

11 A celestial body of arbitrary shape can be approximated by a polyhedron model.

12 Werner [22] developed the closed-form solution of the potential of an arbitrary

13 polyhedron that is split into triangular faces and edges (Fig.1). With this technique,

14 the general formula for the potential can be written as

$$
U(r)=\frac{G \sigma}{2}\left(\sum_{e \in e d g e s} \boldsymbol{r}_{e} \cdot \boldsymbol{E}_{e} \cdot \boldsymbol{r}_{e} L_{e}-\sum_{f \in \text { faces }} \boldsymbol{r}_{f} \cdot \boldsymbol{F}_{f} \cdot \boldsymbol{r}_{f} \omega_{f}\right)
$$

16 where $G$ is the gravitational constant and $\sigma$ is the density of the small body

17 estimated according to the measurements of its component and the classification of

18 its type. The two sums are the contributions from points located on all edges and all

19 faces, respectively, which cover the entire volume of the body. The detailed

20 explanation of all the symbols can be found in Werner and Scheeres [23]. Given a

21 polyhedron model for complex-shape small body, its gravitational potential can be

22 obtained, with relatively high accuracy, even on the surface of the body (in contrast

23 with the spherical harmonics model). However, this approach is computationally

24 heavy and assumes that the body is homogeneous by discarding any macro porosities

25 or large voids.

\footnotetext{
${ }^{1}$ It contains the central body and touches each of the body's vertices.
} 


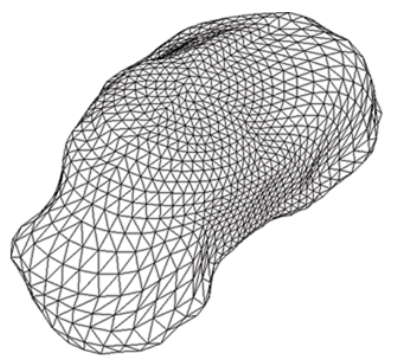

Figure 1 The polyhedron shape model of Itokawa with 3688 faces [24].

3 A similar modeling method is called the 'mass mascon', in which the mass distribution

4 of the body is approximated by a collection of point masses. The mascons were first

5 identified and estimated for the lunar gravitational potential model [25]. The masons

6 model was applied to approximate the irregular gravitational field of asteroid

7 Itokawa for JAXA's Hayabusa mission [26]. This method has relatively low precision

8 at the surface of the small body and it is computationally heavier than the polyhedron

9 model, as shown in Werner's study [23].

10 (C) Geometrical shapes

11 A typical geometry for approximating the shape of an elongated small body is the

12 constant-density ellipsoid. Given an ellipsoid with semi-axes $\alpha>\beta>\gamma$ (Fig.2), its

13 gravitational potential can be expressed as [21]

$$
U_{E}(s)=-\frac{3 G M}{4} \int_{\lambda(s)}^{\infty} \phi(s, v) \frac{d v}{\Delta(v)}
$$

$$
\phi(s, v)=1-\frac{s_{x}{ }^{2}}{\alpha^{2}+v}-\frac{s_{y}{ }^{2}}{\beta^{2}+v}-\frac{s_{z}{ }^{2}}{\gamma^{2}+v}
$$

$$
\Delta(v)=\sqrt{\left(\alpha^{2}+v\right)\left(\beta^{2}+v\right)\left(\gamma^{2}+v\right)}
$$

17 in which $\boldsymbol{s}=\left(s_{x}, s_{y}, s_{z}\right)$ is the vector from the center of the ellipsoid to the particle 18 and $\lambda(\boldsymbol{s})$ is defined as the maximum real root of $\phi(s, \lambda)=0$. The integral can be

19 numerically evaluated with the first and second kind of Carlson's Elliptic Integrals

20 [27]. In analogy to the polyhedron method, this potential is also valid close to and on

21 the surface of the body. Other geometrical shapes cam be used including a straight

22 segment (characterized by its length and mass), two orthogonal segments

23 (characterized by the length and mass of the two segments) and two contact spheres 
1 (characterized by the radius and mass of the two sphere). The detailed descriptions

2 of their potentials can be found in [28, 29, 30], respectively. Moreover, these

3 geometrical shapes can be properly combined to generate more complex shapes and

4 potentials.

5

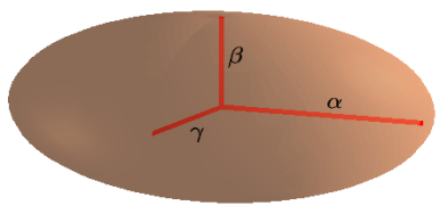

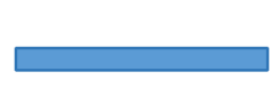
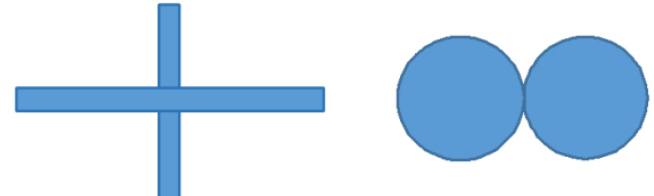

Figure 2 From left to right: an ellipsoid with three semi-axes $\alpha, \beta, \gamma$; a straight segment; two orthogonal segments; two contact spheres.

2.4 The non-gravitational forces

In addition to the irregular gravitational field of the small body, the orbiting particle or s/c is also affected by the perturbations due to solar and planetary gravitational pull, solar radiation pressure (SRP), and outgassing in case of a comet. Planetary perturbations are in general negligible, unless a close encounter between the small body and a planet occurs. In addition, for a large orbital radius, the s/c's motion is less affected by to the perturbations associated with the irregular gravitational field of the object. Strong non-gravitational forces are expected when the small body approaches its perihelion, e.g. due to SRP and outgassing.

\subsubsection{Solar Radiation Pressure}

The SRP perturbation is stronger than the solar gravitation one especially for a s/c with a large area-to-mass ratio, since the surface area is the key element to determine the number of solar photons that interact with the spacecraft, as illustrated in Fig. 3. Systematic study has been performed on its role on s/c's orbital motion around small bodies [1]. If the s/c is far from the small body, SRP can be the main source of perturbation. For sufficiently large-mass small bodies (e.g. asteroid Eros), the perturbation from SRP is very limited. For smaller bodies, with sizes on the order of a few kilometers or less, the SRP can cause the $\mathrm{s} / \mathrm{c}$ to escape or impact on the small body. Generally, the SRP can be modeled in the following three ways. 


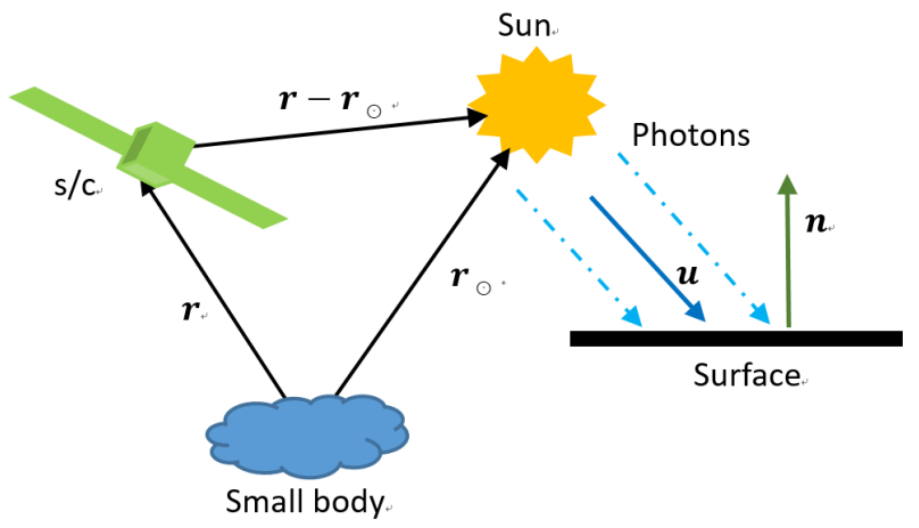

2 Figure 3 Geometry of the s/c orbiting around a small body and the generation of SRP.

4 (a) The cannonball model

5 The cannonball model is the simplest way to model the shape of the s/c, i.e. 6 considering the $\mathrm{s} / \mathrm{c}$ as a sphere, and therefore the s/c's attitude is excluded from the 7 model. Since the directions of vectors $\boldsymbol{n}$ and $\boldsymbol{u}$, representing the normal direction of 8 the surface and the solar radiation direction, respectively, are parallel and the s/c is 9 generally considered as an ideal reflector, the SPR strength is maximum and the 10 corresponding acceleration is given as [31]

$$
\boldsymbol{a}_{S R P}=-\left(1+\kappa_{s}\right) \frac{\rho_{\odot} a_{\odot}^{2}}{c} \cdot \frac{A}{m} \cdot \frac{\boldsymbol{r}-\boldsymbol{r}_{\odot}}{\left\|\boldsymbol{r}-\boldsymbol{r}_{\odot}\right\|^{3}}
$$

12 in which $\kappa_{s}$ and $A / m$ are the albedo and the area-to-mass ratio of the $\mathrm{s} / \mathrm{c}$, 13 respectively; $a=1 \mathrm{AU}$ is the mean distance between Earth and the Sun; $c$ is the 14 light speed in vacuum, $\rho=1367 \mathrm{~W} / \mathrm{m}^{2}$ is the solar flux at $1 \mathrm{AU}$; and $\boldsymbol{r}$ and $\boldsymbol{r}$ are the 15 vector from the small body to the s/c, and from small body to the Sun, respectively.

16 (b) The N-plate model

17 For s/c with larger solar arrays and complex shapes, the N-plate model is applied 18 for a more accurate representation of its shape. This model consists in a collection of 19 flat plates, each of which can have different reflectivity properties. The magnitude of 20 the SRP acceleration varies with the s/c's orientation w.r.t. the Sun and it is given as $21 \quad[32]$ 


$$
\boldsymbol{a}_{S R P}=\frac{\rho_{\odot} a_{\odot}^{2}}{c \cdot m} \cdot \frac{\boldsymbol{r}-\boldsymbol{r}_{\odot}}{\left\|\boldsymbol{r}-\boldsymbol{r}_{\odot}\right\|^{3}} \cdot \sum_{i=1}^{N}\left\{A_{i} \cos \theta_{i}\left[\left(1-\boldsymbol{\kappa}_{s}^{i}\right) \boldsymbol{u}+2\left(\kappa_{s}^{i} \cos \theta_{i}+\frac{\boldsymbol{\kappa}_{\boldsymbol{d}}^{i}}{3}\right) \boldsymbol{n}_{i}\right] H\left(\theta_{i}\right)\right\}
$$

2 where $\cos \theta_{i}=\langle\boldsymbol{n}, \boldsymbol{u}\rangle$ is the dot product of the two vectors; $A_{i}, n_{i},\left(\kappa_{S}^{i}, \kappa_{d}^{i}\right)$ and $H\left(\theta_{i}\right.$

3 ) are the area, normal vector, reflectivity properties and illumination condition for

4 the $\mathrm{i}$-th flat plate, respectively. $H\left(\theta_{i}\right)$ is defined as

$$
H\left(\theta_{i}\right)=\left\{\begin{array}{l}
1, \text { if } \cos \theta_{i}<0 \\
0, \text { if } \cos \theta_{i} \geq 0
\end{array}\right.
$$

6 (c) High-fidelity models

7 To include the auto occultation between different plates, Ziebart [33] proposed to

8 use the Finite Element (FE) technique to determine the illuminated parts of the s/c

9 for its orientation w.r.t. $\boldsymbol{u}$. Specifically, the s/c's structure is firstly defined by simple

10 geometric shapes, which are approximated by the FE distribution of triangles or

11 polygons. Then, a ray is projected in the direction of $\boldsymbol{u}$ and its intersection with the

12 triangles/polygons on the s/c's surface is checked. However, the computational load

13 is heavy for an accurate approximation of the SRP, and it therefore is difficult to apply

14 this modeling approach in real-time.

15 Efforts are made to improve the efficiency of the FE technique. For instance, taking

16 advantage of the periodicity of the SRP force, Fourier series were introduced and

17 applied to develop periodic solutions of the GOLNASS satellite [34] and also to s/c

18 around asteroid [35] and LEO satellite [36]. For s/c on the libration point orbits or

19 the interplanetary trajectories, the attitude profile can be more complex and the

20 Fourier series approach was extended with spherical harmonics for approximating

21 the SRP [37]. The details of these high-fidelity methods are given in the corresponding 22 references.

23 In summary, uncertainties exist in both the modeling of the SRP and the reflectivity 24 property of the $\mathrm{s} / \mathrm{c}$.

\section{$25 \quad 2.4 .2$ Outgassing}

26 Outgassing pressure can bring significant changes in the orbital parameters of the 27 s/c over short time spans. It can be modelled in two different ways: by a continuous 28 model or by discrete jets, which cover the spectrum of the possible outgassing 
environment at a comet [38].

(a) Discrete jets

3 This model assumes that the outgassing field is produced by multiple jets of varying

4 strengths distributed across the surface of the comet [39]. Assuming a jet located at (

$5 r_{j e t}, \lambda_{\text {jet }}, \theta_{\text {jet }}$ ) (in spherical coordinate) on the surface of the comet in its body-fixed

6 frame. A single jet can be described by the half angle $\delta$, the velocity and direction of

7 the outgassing $\boldsymbol{v}_{\text {jet }}$ and $\boldsymbol{e}_{\text {jet }}$, as shown in Fig. 4.

10 With this three dimension characterization, the acceleration from the outgassing jet

11 on the s/c is given as [39]

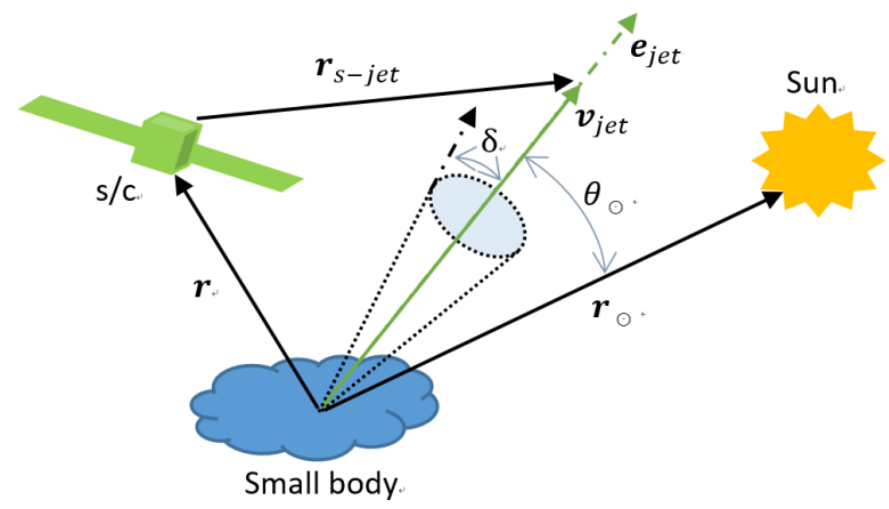

Figure 4 The illustration of outgassing jet on the surface of a comet.

13 in which $A_{j e t}$ is the effective area of the s/c that varies with its orbital parameters
14 since its solar panels are scheduled to point toward the Sun, and

13 in which $A_{j e t}$ is the effective area of the s/c that varies with its
14 since its solar panels are scheduled to point toward the Sun, and

$$
\boldsymbol{a}_{j e t}=\frac{A_{j e t}}{m} \cdot\left(\frac{r_{j e t}}{r_{s-j e t}}\right)^{2} \cdot \boldsymbol{p}_{j e t}
$$

$$
\boldsymbol{p}_{j e t}=Q_{j e t} \cdot v_{j e t} \cdot \boldsymbol{e}_{j e t}
$$

16 is the pressure of the outgassing at the surface of the comet assuming the orientation of the

17 jet at ejection uniformly outwards. The mass ejection rate $Q_{j e t}$ is estimated as [40]

$$
Q_{j e t}=S \cdot Q_{0} \cdot f\left(\theta_{\odot}\right) \cdot g\left(r_{\odot}\right)
$$

19 where $Q_{0}$ is the mass ejection rate with an area equal to the surface area of the comet 20 perpendicular to the Sun at a distance of $1 \mathrm{AU}$, and $S$ is the jet's relative intensity 
1 w.r.t. $Q_{0}$, and $\theta$ is the angle between $\boldsymbol{r}$ and $\boldsymbol{e}_{\text {jet }}$. The functions $f\left(\theta_{\odot}\right)$ and $\mathrm{g}\left(r_{\odot}\right)$

2 depends on the geometry of the jet w.r.t. the Sun and are fully defined in Marsden

3 [41]. Specifically, the mass ejection rate is not constant and varies with the distance

4 from the Sun as well as the change of the surface topological as a result of sublimation,

5 mass loss, or other activities over long time scales.

6 (b) The continuous model

$7 \quad$ The model assumes that the outgassing field is continuous and varies from its

8 maxima at the sub-solar point to a minima at the anti-solar point on the comet. The

9 acceleration from the continuous field outgassing on the s/c can be expressed as [38]

$$
\boldsymbol{a}_{\text {outgassing }}=\frac{A_{\text {jet }}}{m \cdot r^{2}} \cdot[1-\alpha+\alpha \cdot \cos \theta] \cdot \boldsymbol{p}_{\text {jet }}
$$

11 in which $\alpha \leq 0.5$ is the parameter that indicates the outgassing pressure's variation

12 away from the Sun line, i.e. its asymmetry property, and $\theta$ is the angle between the

13 two vectors $\boldsymbol{r}$ and $\boldsymbol{r}$. (You need to add some comments about pros and cons of this)

$14 \quad$ 2.4.3 Thermal effects

15 Similar with the reflection of solar photons of the SRP, the absorption and re-

16 emission of solar energy of the small body also create a tiny thrust, which produces

17 measurable orbital changes over decades and significant orbital effects over millions

18 to billions of years. This is known as the Yarkovshy effect [42]. In addition, this

19 phenomenon also brings about a thermal torque that alters the rotation rate and

20 obliquity of the small body with irregular shape, which is known as Yarkovsky-

21 O'Keefe-Radzievskii-Paddack (YORP) effect [43]. These two effects are illustrated in

22 Fig.5. Their detailed description and discussion can be found in reviews [44] and [45].

23 Specifically, the Yarkovshy force is computed by determining the surface

24 temperature distribution and evaluating the thermal radiation recoil force. It

25 primarily modified the semi-major axis of the small body's heliocentric orbit, and this

26 modification also depends on the physical parameters of the small body, e.g. its

27 obliquity and rotation, size and surface conductivity, etc. In particular, this effect

28 vanishes for both very small and very large bodies. It was first measured from the s/c

29 LAGEOS [46], whose residual along-track acceleration was found to result from the 
1 perturbation of the thermal drag from Earth's emission of infrared radiation due to

2 solar heating. Up to date, the most precise measurement is that of asteroid Bennu, the

3 target of OSIRIS-Rex mission. As the estimation of Bennu's bulk density is well

4 constrained by the Yarkovshy effect, the design challenges of this mission are eased.

$5 \quad$ The YORP effect controls both the long-term evolutions of the small body's spin

6 vector, obliquity and the variation rate of the semi-major axis that is induced by the

7 Yarkovsky effect. Its computation strongly depends on the shape, size, material

8 properties, solar distance and orientation of the small body. If the YORP effect spins

9 up the rotation fast enough, the small body should undergo fission, from which a

10 satellite might be produced. It can be detected by a measurable change of the sidereal

11 rotation of the small body.

12 Since the thermal effects mainly play roles in long timescales evolution, it will not

13 be discussed further in this review that focuses on the orbital motion of s/c motion

14 around a small body in a relatively short time interval. Nevertheless, they are

15 important effects on the orbit and attitude evolutions of the small body.

16

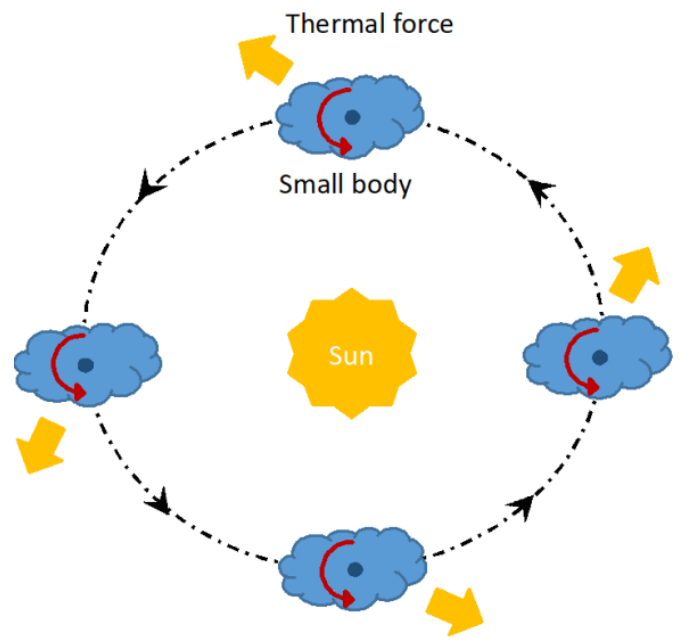

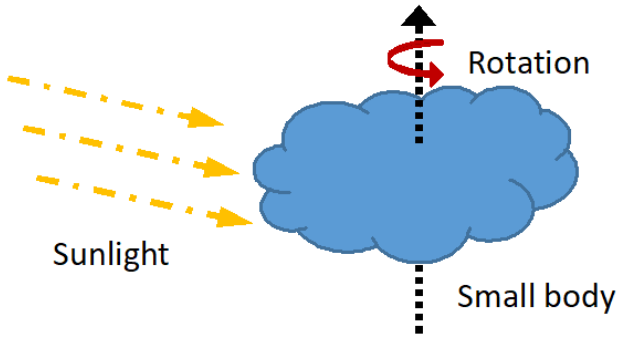

17 Figure 5 The illustrations of the Yarkovshy (left) and YORP (right) effects. For the

18 Yarkovsky effect: the small body's spin axis is perpendicular to the orbital plane. A

19 fraction of the solar insolation is absorbed only to later be radiated away, yielding a

20 net thermal force in the direction of the wide arrows. For the YORP effect: an

21 asymmetrical small body absorbs the sunlight falling on it and then reemits the

22 energy in the infrared as thermal radiation in different directions. A net torque is 
therefore generated that spins up the body.

$4 \quad$ Three approaches are generally followed to design orbits around small bodies. The

5 first one consists in applying the Lagrange Planetary Equations (LPE) [20], which

6 describes the influence of the perturbing forces on the variations of the osculating

7 orbital elements. Generally, the LPE is averaged and the secular evolution of the

8 dynamics is obtained, and the frozen orbits, when orbital elements stay constant on

9 average or suffer secular motion, are identified. Studies using this method can be

10 found in [47, 48]. In the second approach the design is carried out without resorting

11 to averaging. Equilibrium points (EPs) of the full dynamical system, expressed by a

12 set of ordinary differential equations (ODEs) in which the relevant perturbations are

13 included, can be obtained in most cases by solving for the zeros of the body-fixed

14 velocity and acceleration of the s/c. Families of periodic and quasi-periodic orbits can

15 be found around these EPs by applying numerical continuation and correction

16 methods. This approach was followed in $[1,49,50]$. The third method consists in

17 applying a pure numerical procedure for direct search and continuation of periodic

18 orbits and of stable motion around the small body without the transformation of the

19 dynamics. The related research can be found in $[51,52,53]$. In general, the first two

20 approaches are applied for preliminary analysis and design of mission orbits. And the

21 last one is mainly for detailed characterization of the phase space of orbital dynamics

22 in a more specific and accurate irregular gravity field.

23 The design of the operation orbit is strongly affected by the type of perturbations 24 that are included. Different perturbations are affected by a different level of 25 uncertainties, and this uncertainty quantification plays a key role in trajectory design 26 about small bodies.

\section{$27 \quad 3$ The classification of uncertainties}

\subsection{Uncertainties from gravitational forces}

29 In addition to the state uncertainty from navigation and orbit determination (OD) 30 errors, our knowledge of the physical parameters of a small body is affected by large 
1 uncertainties at least during the first phase of the rendezvous. During the following-

2 up approaching and orbiting mission phases, these uncertainties can be reduced and,

3 e.g., the $3^{\text {rd }}$ and $4^{\text {th }}$ order gravitational harmonics can be determined with good

4 accuracy. However, due to the contamination from possible control forces or physical

5 forces that are not well modeled (e.g., the outgassing around comets), these

6 parameters are still not completely determined. These uncertainties consequently

7 restrict the capability of OD, orbit prediction and construction, which in turn limit the

8 scientific products [54].

93.2 Uncertainties from non-gravitational forces/ (Errors of the estimation of these 10 parameters)

11 Other stochastic non-gravitational forces, such as the SRP, the outgassing, gas leaks

12 from attitude control system, thruster imbalance, affect the s/c motion. These forces

13 are generally several orders of magnitude smaller than gravity, and can be divided

14 into constant and time-varying contributions. The first ones can be modeled as biased

15 parameters. The latter ones can be split into rapidly varying forces, which tend to

16 average out over time, and forces with frequencies commensurate with the s/c orbital

17 period which can result into resonances and are therefore troublesome [55].

18 During the descent phase of landing missions, the physical parameters of the small

19 body are better constrained from iterations of previous phases. Nevertheless, the

20 motion in extremely close proximity is highly unstable due to the strong

21 perturbations from the body irregular gravity field and outgassing (for comets).

22 Therefore, small uncertainties in the s/c's state and maneuvers/executions can cause

23 a wide distribution of the propagated state. As a result, the braking maneuver for

24 descending is usually executed before the one planned with deterministic dynamics

25 to give enough time for the (autonomous) guidance, navigation and control (GNC)

26 system to counteract these uncertainties. Otherwise, the s/c might impact on the

27 small body surface even before the maneuver is performed [56].

28 To some extent, these uncertainties will impact on choosing landing strategies. For 29 asteroids with small masses, it is more appropriate to 'land' through hovering or TAG,

30 which, for instance, is applied to both ORISIS-Rex and Hayabusha2 missions. This is 
1 due to the fact that the orbital motion of the $\mathrm{s} / \mathrm{c}$ is more sensitive to these

2 uncertainties given the weak gravity attraction and more control efforts might be

3 needed to counteract them. The other option is hard landing, i.e. a lander is delivered

4 and stays on the surface of the asteroid for longer-time sample collections and more

5 detailed (even on-board) analysis, for instance, the Rosetta mission and DART. This

6 can be explained by the fact that the orbital motion of the s/c has larger resistance to

7 these uncertainties in general under stronger gravity field.

83.3 Aleatory and epistemic uncertainties

9 Uncertainties can be primarily divided into two categories: aleatory and epistemic 10 uncertainties [57]. The former results from the random nature of the system or input 11 data, while the latter one is always related to incomplete modeling of the system 12 because of a lack of knowledge. The uncertainty in the initial state, gravity field, SRP, 13 outgassing are mainly treated as aleatory uncertainties, with distribution properly 14 modeled by probability density function (PDF) [58]. The uncertainties related to the 15 gas leak, thruster imbalance and parameters of spacecraft's sub-systems are 16 considered as epistemic. They can be appropriately represented with Evidence 17 Theory [59], which is an alternative approach to the traditional probabilistic 18 representation. Rather than assuming on the probabilities or system margins, this 19 theory combines evidence and information from multiple sources and models the 20 conflicts among them, for crystallizing the inherent uncertainties [60]. This approach 21 helps formulating the mission design process into an optimization problem. For its 22 detailed application in robust engineering design, and especially for space systems 23 and space trajectories, the reader can refer to $[8,61,62,63]$. Nevertheless, this survey 24 mainly focuses on the aleatory part of the uncertainties.

\subsection{Example-NEAR mission}

26 For an intuitive awareness of the state, gravitational and non-gravitational 27 uncertainties, as an example, Tables $1 \mathrm{a}, 1 \mathrm{~b}$ and $1 \mathrm{c}$ give their quantitative information 28 from NEAR mission to Eros [55]. The OD error is described in the orbital frame of the $29 \mathrm{~s} / \mathrm{c}$, and the radial, along-track and cross-track directions correspond to the $\mathrm{x}^{-}, \mathrm{y}^{-}$ 30 and z-axis, respectively, as illustrated in Fig.6. For different orbit radius, the 
1 maximum and minimum errors are along the along-track and cross-track directions,

2 respectively. Moreover, the closer the s/c is to Eros, the more accurate the s/c's orbit

3 can be determined, due to the increasing strength of the Doppler data or the

4 improving capability of optical navigation. From the estimations of the orbit and

5 attitude of the s/c, the physical properties of Eros can be determined with a certain

6 accuracy level. The $1 \sigma$ uncertainty of the SRP coefficient is up to about $10 \%$, and that

7 of the gas leak (at the order of $10^{-12}$ ) is much smaller by comparing with the GMvalue

8 (at the order of $10^{-4}$ ). The small body's internal structure and mass distribution can

9 be characterized from the determination of the shape and gravity harmonics. The 10 errors of the harmonic terms $C_{20}, C_{22}, C_{30}, C_{40}$, reduce by about one order of 11 magnitude when the s/c orbit radius reduced from $200 \mathrm{~km}$ to $35 \mathrm{~km}$. Nevertheless, 12 the relative errors generally increase rapidly for higher degree harmonics, as it has

13 been observed by comparing the uncertainties of the second, third and fourth order 14 harmonics respectively. The parameters $M$ and $G M$ can be determined with good 15 precision at the error of $10^{-3}$. One interesting phenomenon is that their estimation is 16 more accurate from orbit radius of $200 \mathrm{~km}$ than from orbits of $50 \mathrm{~km}$ and $35 \mathrm{~km}$.

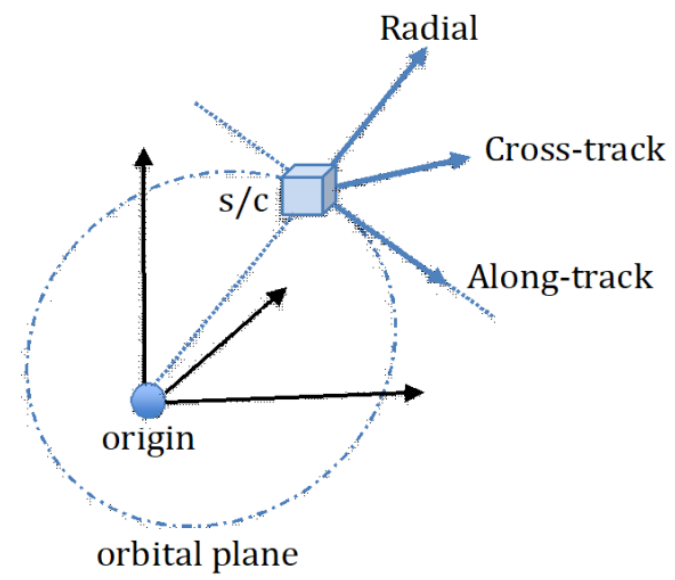

18 Figure 6 The radial, along-track and cross-track directions in the orbital frame of the $19 \mathrm{~s} / \mathrm{c}$., where $\mathrm{x}$-axis is in the radial direction and $\mathrm{z}$-axis is in the direction of the angular 20 momentum of the orbit and y-axis completes the right-handed reference frame. 


\begin{tabular}{lccc}
\hline Orbit Radius/km & Radial/m & Downtrack/m & Crosstrack/m \\
\hline 200 & 856 & 1470 & 117 \\
50 & 29 & 95 & 4.9 \\
35 & 15 & 54 & 1.6 \\
\hline
\end{tabular}

Table $1 \mathrm{~b}$ Non-gravitational force parameters and their uncertainties [55]

\begin{tabular}{lll}
\hline Parameters & Nominal Values & Error $(1-\sigma)$ \\
\hline SRP & & \\
momentum transfer coefficient & 1.5 & 0.15 \\
Gas leak & & \\
constant bias $\left(\mathrm{km} / \mathrm{s}^{2}\right)$ & 0 & $5.0 \times 10^{-12}$ \\
variable modeling error $\left(\mathrm{km} / \mathrm{s}^{2}\right)$ & 0 & $5.0 \times 10^{-13}$ \\
\hline
\end{tabular}

Table 1c Eros gravitational estimation errors [55]

\begin{tabular}{lllll}
\hline Parameters & Nominal Values & $200 \mathrm{~km}(1-\sigma)$ & $50 \mathrm{~km}(1-\sigma)$ & $35 \mathrm{~km}(1-\sigma)$ \\
\hline Mass Properties & & & & \\
$M(\mathrm{~kg})$ & $1.330 \times 10^{16}$ & $1.2 \times 10^{13}$ & $3.8 \times 10^{13}$ & $1.8 \times 10^{13}$ \\
$G M\left(\mathrm{~km}^{3} / \mathrm{s}^{2}\right)$ & $8.865 \times 10^{-4}$ & $7.5 \times 10^{-7}$ & $2.5 \times 10^{-6}$ & $1.2 \times 10^{-6}$ \\
Gravity Harmonics & & & \\
$C_{20}$ & $-3.03 \times 10^{-2}$ & $1.3 \times 10^{-2}$ & $9.2 \times 10^{-3}$ & $1.8 \times 10^{-3}$ \\
$C_{22}$ & $+3.78 \times 10^{-2}$ & $1.4 \times 10^{-2}$ & $6.5 \times 10^{-4}$ & $1.8 \times 10^{-4}$ \\
$C_{30}$ & $+1.05 \times 10^{-4}$ & $5.2 \times 10^{-2}$ & $2.2 \times 10^{-4}$ & $2.8 \times 10^{-5}$ \\
$C_{40}$ & $+4.09 \times 10^{-3}$ & $3.8 \times 10^{-1}$ & $1.6 \times 10^{-2}$ & $1.5 \times 10^{-3}$ \\
\hline
\end{tabular}

54 The current research status of uncertainty analysis

6 For space missions, the primary objective of uncertainty analysis is to identify the

7 influences of different uncertainties on the orbital motion for robust mission design.

8 The objective of uncertainty propagation is to estimate the distribution of state $\boldsymbol{x}$

9 due to the presence of the uncertainties, from both the initial state and system 
1 parameters. Denoting the PDF distribution of state $\boldsymbol{x}$ as $p_{x}(\boldsymbol{x}, t)$, the distribution of

$2 \quad \boldsymbol{x}$ in space $\boldsymbol{S}$ can be obtained as [57]

$$
P(\boldsymbol{x} \in \boldsymbol{S})=\int_{\boldsymbol{S}} p_{x}(\boldsymbol{x}, t) d \boldsymbol{x},
$$

4 and this integration over the whole state space is one. The time evolution of $p_{x}(\boldsymbol{x}, t)$

5 can be obtained by writing down and solving the so-called Fokker-Plank differential

6 Equation (FPE) [64]. However, since in orbital mechanics the dimension of the state

7 is high (at least 6 dimensions) and the dynamics is nonlinear (especially for motion

8 around irregular small bodies), it is difficult to solve the FPE directly and completely.

9 Therefore, more practical techniques have been developed over the years to deal with

10 the challenges of uncertainty propagation. In this context, three main categories, i.e.

11 the Monte Carlo (MC) method, the linear method and the nonlinear method, can be

12 identified. Their basic features are described in the following.

134.1 The uncertainty propagation algorithms

14 4.1.1 The Monte Carlo and linear method

15 (a) MC method

16 MC is a sample-based method [65]. Given the initial distribution of the uncertain

17 variables $\boldsymbol{s}$ as $p_{s}\left(\boldsymbol{s}_{0}, t_{0}\right)$, a number $N$ of random samples can be generated according

18 to this distribution. In fact, $s$ can either be the state $x$ or parameters of the

19 dynamics, saying $\boldsymbol{q}$, or a combination of $\boldsymbol{x}$ and $\boldsymbol{q}$. Therefore, the propagations of the

20 dynamics are either with $N$ initial states $\boldsymbol{x}_{i}(i=1,2, \ldots N)$ and the same parameter $\boldsymbol{q}_{0}$,

21 or with the same initial state $\boldsymbol{x}_{0}$ but $N$ initial parameters $\boldsymbol{q}_{i}(i=1,2, \ldots N)$, or with

22 the same variables of $x$ and $\boldsymbol{q}$ that are certain and $N$ samples of the uncertain

23 variables of $x$ and $q$. Anyhow, the $N$ final states are statistically analyzed, from

24 which the statistical moments (e.g. the mean and the covariance matrix) of the

25 mapped quantities of interest are obtained. The advantage of MC is that it is easy to

26 implement and can propagate with the full non-linear dynamics and the non-Gaussian

27 PDF of the initial uncertainties of either states or parameters. Generally, its precision

28 increases with the increasing number of samples, i.e., the final PDF approaches its

29 true value if the sample number $N$ goes to infinity. The main drawback of MC is that

30 it is computationally expensive, especially for a large number of samples and long 
1 propagation time, due to its sample-based characteristic.

2 (b) Linear method

3 Different from the MC method, the linear method mainly deals with dynamics with

4 linearization, i.e. representing the nonlinear dynamics with its linearized model that

5 approximates the dynamics of a neighboring motion w.r.t. the nominal motion using

6 first-order Taylor expansions along this nominal motion [66]. Therefore, it is an

7 analytical way that propagates the initial uncertainties using the state transition

8 matrix (STM). Without generating samples, the mean and the covariance matrix can

9 be computed directly. In particular, if the mean is zero, the covariance is obtained by

10 mapping the initial covariance through the STM. However, for the uncertainty of the

11 parameter, this method is not a straightforward way unless the Taylor expansion is

12 w.r.t. the uncertain parameters that are mapped into the final state through properly

13 augmenting the STM matrix. The accuracy of the linear method reduces for a highly

14 nonlinear system and for variables with large uncertainties, because of its essentiality

15 of linearizing the dynamics.

16 As a result of the advantages and disadvantages of the MC and the linear method,

17 many analytical and semi-analytical nonlinear methods have been developed in the

18 attempt to achieve a better compromise between efficiency and accuracy. The most

19 relevant ones are reviewed in the next section.

20 4.1.2 Analytical and semi-analytical nonlinear methods

21 Based on either inherent dynamics or sample approximations, the nonlinear methods

22 are capable of characterizing the nonlinear and non-Gaussian uncertainty

23 propagation. Detailed mathematical definitions and descriptions of these methods

24 and their applications in SSA are covered in Luo's review [12]. The characterizations

25 of several typical methods are summarized as follows.

26 (a) Differential Algebra

27 With the Differential Algebra (DA) technique the flow of a set of ordinary

28 differential equations (ODE) can be expanded in Taylor series w.r.t either the initial

29 condition or system parameter [67]. By implementing the algebra of multivariate

30 Taylor polynomials, this expansion is obtained in an automatic way up to an arbitrary 
1 order without the need to write variational equations. The DA framework

2 incorporates the algebraic, differentiation and integration operators. The availability

3 of the Taylor approximation of the flow allows performing statistical analyses either

4 by using samples in a Monte Carlo fashion [ref Apophis], by computing high order

5 statistical moments (Valli paper Journal of guidance), or by directly mapping PDF

6 (Armellin Di Lizia probabilistic IOD Journal of guidance). DA has been applied to

7 asteroid encounter analysis [68], orbit conjunction analysis [69], s/c navigation and

8 guidance algorithms [70], optimal control strategies [71], etc.

9 (b) State Transition Tensors

10 Uncertainty propagation by means of State Transition Tensors (STT), proposed by

11 Park and Scheeres [72], share many similarities with the DA-based approach as it is

12 also a semi-analytical method that approximates the flow of the dynamics with high-

13 order Taylor series expansions. The main difference resides in the way in which the

14 Taylor polynomials are computed. In particular, the coefficient of the SST are

15 computed by writing and integrating higher order variational equations along with

16 along the nominal trajectory. For this reason, the SST methodology is typically less

17 efficient than the DA approach, resulting in lower order expansions. Once the SST are

18 obtained by the same approaches, the mapped statistical moments can be obtained

19 either by evaluating samples or applying analytical formulae for specific initial

20 distributions. For instance, the mean and the covariance matrix at any time are

21 obtained as an algebra operation given their initial conditions.

22 For its application, Park [73] discovered that the secular variations, rather than the 23 short-period effects, in the dynamical model are dominant in the accuracy of 24 uncertainty propagation. Considering the atmospheric drag force, Fujimoto [74]

25 developed an analytical nonlinear uncertainty propagator for both conservative and 26 non-conservative dynamical systems.

27 (C) Unscented Transformation

28 Unscented Transformation (UT) was proposed by Julier [75]. It is based on the idea 29 that approximating the probability distribution might be easier than approximating 30 the nonlinear dynamics. UT approximates this distribution by nonlinearly 
1 propagating a few samples, which are a set of weighted sigma-points chosen to 2 capture the mean and the covariance matrix of the initial distribution. The samples 3 are propagated with the nonlinear dynamics to yield the transformed sigma-points,

4 from which the transformed mean and covariance matrix are obtained. The 5 symmetric (extended) set method [76] is usually employed to generate samples

6 (rather than random ones) and determine the associated weights that are not 7 restricted to the range [0,1]. Moreover, the number of required samples is $2 N+1(N$ 8 is the dimension of the nonlinear system), which is much smaller than that required 9 for MC, indicating its computational efficiency especially for low-dimensional system.

10 (d) Polynomial Chaos

11 The Polynomial Chaos Expansions (PC) method was first proposed by Wiener [77].

12 PC is also a semi-analytical method that approximates both the input and output 13 uncertainties of a system with series expansions in random variables. Given the 14 probability measure of the initial input, the output uncertainty can be expressed by 15 the weighted summation of orthogonal polynomial chaos that is constructed in these 16 random variables. The weighted coefficients are computed through intrusive and 17 non-intrusive methods, from which the mean and the covariance of the output 18 uncertainties can be directly obtained. This method is fast and efficient, especially for 19 dealing with the fully non-linear dynamics and for the uncertainties that are non20 Gaussian and are difficult to be represented by PDF. PC has been applied to satellite 21 collision probability estimation [78]. Moreover, considering uncertainties in both 22 states and system parameters, the PC method demonstrated its great advantage in 23 identifying uncertainty evolution for hypersonic dynamics [79].

24 In summary, these four methods have been mainly used to analyze space missions 25 around Earth, in terms of guidance, navigation and control, etc. Nevertheless, their 26 applications in characterizing the impact of uncertainties on small body mission

27 design and analysis are limited. To address this problem, the uncertainty problems 28 and studies in the previous and ongoing small body missions are therefore presented 29 and summarized in the next section. 
2 In this section, the several uncertainty studies in previous and ongoing small body

3 missions are presented and summarized.

4 (a) Hayabusa and Hayabusa-2 missions

5 For a small body with relatively large mass, the s/c is capable of circling around the

6 body for long free motion arcs and the telemetry data is used to estimate the small

7 bodies' gravity fields, e.g. Eros [80] and Vesta [81]. However, Hayabusa's target

8 asteroid Itokawa is highly elongated and has a very weak gravity field (i.e. relatively

9 small mass). The s/c thus performed hovering over the asteroid, for both scientific

10 observations and gravity estimation [82]. During JAXA's Hayabusa's close proximity

11 to Itokawa, its gravity was also determined with uncertainties (Table 2). However, for

12 the small-mass Itokawa, the orbital motion was highly unstable as the perturbation

13 from SRP is relatively large. Therefore, the mission applied the so-called solar

14 terminator orbit (STO) [1], which is perpendicular to the Sun-asteroid line and quasi-

15 stable if the SRP is dominant. For both cycling orbit and STO, Melman [83] quantified

16 the impacts of gravity uncertainty on the evolution of them with the MC method. The

17 motion was found to be more sensitive to the physical uncertainties of the asteroid

18 with weaker gravity field, and for motions in mean-motion resonances ${ }^{2}$ with the

19 asteroid's rotation. These findings assist the mission planners in assessing the posed

20 risk and designing appropriate orbits.

21 Launched in 2014, Hayabusa2 arrived at its target asteroid Ryugu in June 2018.

22 Comparing with Itokawa, Ryugu has a more regular shape (diamond shaped) and also

23 has a weak gravity field with many rubble piles. First the s/c hovered at an altitude

24 about $20 \mathrm{~km}$ [84] (also called the home position), to map the asteroid for its spin

25 orientation, rotation period, shape, obliquity and topography. Following that, the s/c

26 performed several ballistic descents vertically to the altitude at $5 \sim 6 \mathrm{~km}$, to

27 sufficiently measuring the GM of Ryugu, during which the SRP coefficients were also

28 estimated. However, the mass was determined still with an uncertainty of 1.3\%, ${ }^{2}$ The mean motion rate of the $\mathrm{s} / \mathrm{c}$ is in commensurability with the rotation rate of the small
body. 
1 mainly due to the uncertainties of the SRP on the s/c [84]. These operations 2 confirmed previous studies that the longer the free motion time and the lower the 3 minimum altitude bound are, the better the GM can be constrained [85]. And this 4 information is critical for planning the succeeding mission sequence, selecting the 5 potential sampling site and deploying the lander and rovers [86]. After measuring the 6 gravity, the s/c delivered the first rover MINERVA-II, which hopped among different 7 locations for science operations and sent back images of the surface of Ryugu with 8 small boulders. Then the lander MASCOT was deployed at the altitude of $51 \mathrm{~m}$ and 9 obtained much clearer images of the surface, which assisted selecting the sampling 10 site. During these phases, three touchdown rehearsals were performed and the target 11 marker was released for the touchdown location. Then, the s/c was inserted to the 12 'conjunction trajectory' for the solar conjunction phase, during which small insertion 13 errors might result in an undesired close approach or collision with Ryugu. Moreover, 14 using observation data during this phase, Ryugu's orbit was recalculated, which 15 allows the s/c maintain the home position more stably. The s/c completed the first 16 touchdown on the surface of Ryugu in Feb. 2019, collecting samples and returning 17 back to the home position, which requires very precise navigation and guidance. In 18 April, the s/c released an impactor on the asteroid and created an artificial crater. The 19 formation of the crater, the dispersion of the ejectors and the internal structure will 20 be observed and examined in detail. The second touchdown operation that is planned 21 in May will collect the material in the crater. The second rover MINERVA-II is 22 scheduled to be deployed in July. The s/c will depart from Ryugu at the end of 2019

23 [87]. It can be noticed that the mission operations are very complicated and require 24 high autonomy of the s/c to achieve mission objectives.

25 (b) Rosetta mission

26 Launched in 2004, Rosetta arrived at its target comet 67P/Churyumov27 Gerasimenko (denoted as 67P) in August 2014. During its ten-year space travel, it 28 flew by two asteroids, i.e. 2867 Šteins in 2008 and 21 Lutetia in 2010. On arrival, the $29 \mathrm{~s} / \mathrm{c}$ 's orbiting around the comet was found to be much more difficult than expected 30 because of its unexpected double-lobed structure separated by a narrow neck [88]. 
1 After having been captured by the weak gravity of 67P, the s/c orbited the comet 2 and gathered data to characterize the environment and the comet nucleus. The mass 3 and gravity field of the comet were derived from the measured velocity perturbations 4 of the s/c at distances between 10 and $100 \mathrm{~km}$. The higher order of the gravity could 5 already be sensed and determined at distances below $30 \mathrm{~km}$, due to the odd shape of 6 the nucleus. The values of mass and gravity field up to degree and order two are given 7 in Table 2 [89]. Compared with the GM solution for the point mass at a distance larger 8 than $30 \mathrm{~km}$, there is a large error in the GM solution from the tracking data between $910 \mathrm{~km}$ and $20 \mathrm{~km}$, due to the outgassing perturbation, although it is very weak.

10 In addition to the tracking data, with the assumption of constant density and based 11 on the polyhedron shape models of 67P from LAM and DLR, the gravity harmonics 12 can also be determined and are given in Table 2. It can be seen that the $\mathrm{C}_{20}$ and $\mathrm{C}_{22}$ 13 terms from the three different efforts agree well within a 3- $\sigma$ standard deviation. 14 Moreover, a sensitivity analysis about the impact of the shape error on the estimated 15 harmonics was carried out and it was found that the shape-induced error was less 16 than $1 \%$ if the positioning error of the vertices of the polyhedron model is less than $17140 \mathrm{~m}$ [90]. During the mission, a change of the rotation rate of 67P due to the torques 18 of solar gravitational pull and outgassing was observed. It was discovered that 19 outgassing is responsible for the majority of rotational changes and the 20 corresponding uncertainties.

21 After a few months after arrival, the s/c deployed the lander Philae in November

22 2014. Though it failed to land safely on the planned region and instead bounced to a 23 poorly illuminated region, Philae is the first lander on a comet ever and returned 24 unique science data. Then, Rosetta accompanied the comet through its perihelion and 25 through the comet's travel towards the orbit of Jupiter.

26 During the comet's perihelion passage by the Sun in August 2015, the amount of 27 water vapour released by the comet increased by a factor of ten. In addition, large jets 28 from the neck region and clouds of dust and gas (known as the coma) were observed. 29 Moreover, the plume was seen by the s/c in July 2016, as the comet was heading away 30 from the Sun at a distance of almost 500 million km. Alongside a steep increase in the 
1 number of dust particles flowing from the comet, Rosetta also detected tiny grains of

2 water-ice. In addition to the ice evaporation in the sunlight, other more energetic

3 processes were believed to occur to fling that amount of dust into space. Uncovering

4 these mechanism is still a great challenge.

5

6 Table 2 Values of GM and the $2^{\text {nd }}$ order gravity coefficients of $67 \mathrm{P}$ with 1 - $\sigma$

$7 \quad$ uncertainties from the tracking data, LAM and DLR shape models [89].

\begin{tabular}{cccc}
\hline Parameter & \multicolumn{2}{c}{ Tracking $>30 \mathrm{~km}$} & Tracking $>10 \mathrm{~km}$ \\
\hline $\mathrm{GM}$ & $666.2 \pm 0.2$ & & $666.1 \pm 0.4$ \\
\hline Coefficients & Tracking data & LAM model & DLR model \\
\hline $\mathrm{C}_{20}$ & $-0.035 \pm 0.002$ & $-0.033 \pm 0.001$ & $-0.037 \pm 0.001$ \\
$\mathrm{C}_{21}$ & $-0.0001 \pm 0.0003$ & $0.0024 \pm 0.0001$ & $0.003 \pm 0.0001$ \\
$\mathrm{C}_{22}$ & $0.045 \pm 0.001$ & $0.0444 \pm 0.0004$ & $0.045 \pm 0.0004$ \\
$\mathrm{~S}_{21}$ & $0.0006 \pm 0.0008$ & $-0.0012 \pm 0.0001$ & $-0.0009 \pm 0.0001$ \\
$\mathrm{~S}_{22}$ & $-0.0006 \pm 0.0009$ & $-0.0007 \pm 0.0001$ & $0.0006 \pm 0.0001$ \\
\hline
\end{tabular}

9 (c) The OSIRIS-Rex mission

10 After launch in 2016, NASA's first asteroid sample return mission, OSIRIS-Rex, 11 entered orbit around its target asteroid Bennu on December $3^{\text {rd }}$, 2018, setting the two

12 records of orbiting the smallest body ever with the closest distance ever. On arrival,

13 the s/c flew over the polar and equatorial regions of Bennu, to improve the estimates

14 of its mass and spin rate and to obtain a more precise shape model, which in turn

15 refined the follow-on trajectory design. The preliminary characterization of Bennu's

16 mass and GM are given in Table 3 [91].

17 After arrival, the s/c had been orbiting Bennu for about one month at an altitude

18 between $1.6 \mathrm{~km}$ and $2.1 \mathrm{~km}$, which is the navigation campaign phase (orbital A phase)

19 that aims to transform the stellar optical navigation to the landmark optical

20 navigation and to gain experience of navigating in close proximity to a small body.

21 During this phase, small particles near Bennu were detected and determined not to

22 pose risk to the s/c. After that, the s/c began the detailed survey phase to map the 
1 global properties of Bennu and select the candidate sample sites, which will be

2 narrowed down during the follow-up orbital B phase when the s/c orbits around

3 Bennu the closest ever with the radius of $1 \mathrm{~km}$ and performs radio science

4 experiments [92]. As the gravity of Bennu is so weak, forces of the SRP and the

5 thermal pressure from Bennu's surface (i.e. the Yarkovshy effect on the s/c) become

6 much more relevant and can perturb the s/c in its orbit. Therefore, STO was chosen

7 to be the nominal science orbit. Sensitivities of the terminator orbits to the maneuver

8 execution errors were analyzed based on the averaged dynamics and tested against

9 MC simulations. The frozen-STO, which is fixed in the Sun-asteroid rotating frame, is

10 found to be more robust against the execution errors [93].

11 After a few planned rehearsals, the sample will be retrieved from the asteroid 12 during the touch and go phase (TAG) [94], after which the s/c will drift away from

13 Bennu to a safe distance and stay a longer time until 2023, for characterizing the

14 effects of YORP and Yarkovsky on Bennu's rotational and orbital motion, respectively.

15 The rotation rate of Bennu is estimated to accelerate continuously at $3.63 \pm 0.52 \times$

$1610^{-6}$ degree/day ${ }^{2}$, probably due to the YORP effect [95]. Considering errors from

17 measurement noise, ground station location, navigation, maneuver executions,

18 asteroid's ephemeris, gravity modeling and SRP, the position and velocity were 19 accurately estimated with accuracies at meter and centimeter per second levels,

20 respectively (Table 3b) [94]. These small navigation errors were due to more 21 accurate restriction of the small body's parameters in the final landing phase. Taking

22 into account these uncertainties and with the assumption of Gaussian distribution, a

23 TAG strategy with a closed-loop control algorithm was developed. Its robustness was

24 assessed with $\mathrm{MC}$ analysis, and it was found to meet the requirement of delivering the

$25 \mathrm{~s} / \mathrm{c}$ to within $25 \mathrm{~m}$ of a given TAG site with about $3 \sigma$ uncertainty. However, the

26 predetermined TAG design might be refined and changed due to data gathered from

27 Bennu since arrival.

28 Table 3a. The Bennu bulk density and related quantities with 1- $\sigma$ uncertainties [91]

\begin{tabular}{ll}
\hline Bulk density $\boldsymbol{\rho}\left(\mathrm{kg} / \mathrm{m}^{3}\right)$ & $1190 \pm 13$ \\
Mass M $\left(10^{10} \mathrm{~kg}\right)$ & $7.329 \pm 0.009$
\end{tabular}


$\mathrm{GM}\left(\mathrm{m}^{3} / \mathrm{s}^{2}\right) \quad 4.892 \pm 0.006$

1 Table 3b. Navigation uncertainty (3- $\sigma)$ [94]

\begin{tabular}{c|c|c|c|c|c|c}
\hline \multirow{2}{*}{} & \multicolumn{3}{|c|}{ Position uncertainty $(\mathrm{m})$} & \multicolumn{3}{c}{ Velocity uncertainty $(\mathrm{m} / \mathrm{s})$} \\
\cline { 2 - 7 } & radial & along-track & cross-track & radial & along-track & cross-track \\
\hline Nominal GM & 0.529 & 3.132 & 0.633 & 0.173 & 0.035 & 0.077 \\
\hline
\end{tabular}

2 (d) AIDA mission

3 The Asteroid Impact and Deflection Assessment (AIDA), consisting of NASA's

4 Double Asteroid Redirection Test (DART) mission and ESA's Asteroid Impact Mission

5 (AIM), aims to demonstrate the kinetic impactor deflection technique. DART will

6 impact the secondary of the binary asteroid Didymos. AIM is the s/c that is designed

7 to observe the whole impact process and the binary system, and it will release two

8 CubSats for additional scientific observations [96]. Unfortunately, AIM did not go into

9 a further study case and is now simplified as Hera mission in the study case [97],

10 while DART is planned to launch in 2021 [98]. Reliability analyses of uncertainties on

11 both orbiting and landing phases have been performed during the preliminary

12 mission design.

13 For the orbiting phase, the parameters of the asteroid, e.g. mass, shape, rotation, 14 and the s/c's mass, surface area and reflectivity, were varied to evaluate their

15 influences on the stability and lifespan of orbital motion in the equatorial plane of the

16 asteroid [99]. Since the duration of most small body missions is about several months

17 (that is much shorter than that of Earth missions) for practical purpose, the stability

18 is therefore characterized as the orbit's duration of free motion arcs (without

19 maneuvers) for radio or laser tracking and navigation [99]. By using MC simulations,

20 retrograde orbits were found to allow about 90 days' free motion arcs. Moreover,

21 during the landing phase, the separation error of the deployment of the CubeSats was

22 found to dominate the uncertainties of their GNC errors and the density of the

23 secondary body as well as the landing precision [100].

24 In summary, the uncertain gravity field of the small bodies and the execution errors

25 were found to have obvious impacts on orbital motions. Moreover, almost all of the 
1 studies were made by applying the MC method, which is time-consuming and not

2 appropriate for autonomous navigation and onboard calculations. Therefore, more

3 efficient methods have to be introduced and explored to approximate the results of

4 MC simulations with the required accuracy. The advantages and drawbacks of

5 applying the nonlinear methods, described in Section 3.1.2, to small body

6 explorations will be addressed in the following section.

$7 \quad 5$ The prospects of future research

8 To meet challenges from new mission scenarios, two main aspects can be addressed:

9 the complete modeling of the uncertain dynamics, and the application of new

10 methods. Both of them are discussed in this section.

\subsection{Complete modelling}

12 For orbital motion around asteroids, to accurately determine the forces and 13 perturbations on the $\mathrm{s} / \mathrm{c}$, the knowledge of the asteroid needs to be improved, in

14 terms of its shape, density, rotation state, mass ejection, etc. However, these 15 parameters might be changed due to the Yarkowsky and YORP effects during a long 16 time period. Therefore, for very long-duration missions, this problem should be 17 considered and managed as model uncertainties.

18 Specifically, the uncertainty of the rotation state of the small body has a great 19 influence on the detailed mission planning, as mentioned in Section 2.2. Thus, during 20 the proximity mission operations it should be included in the uncertainty modeling 21 and its influence should be characterized in detail for robust mission design. For 22 comets, the outgassing is a significant perturbation on its nearby orbital motion, and 23 it becomes stronger during the comet's perihelion phase. However, it is difficult to 24 predict due to the insufficient knowledge about the material properties and its 25 injecting speed, etc. Moreover, similar with that of SRP, the perturbation of the 26 outgassing also relates to the surface area of the s/c that the outgassing force acts on.

27 The inclusion of it in the modeling and its investigation is highly required, because it 28 can contribute to a more robust mission design.

29 In addition, as the altitude of the s/c increases, the magnitude of the SRP force 30 becomes large and comparable with that of the gravity force $[1,101]$. Therefore, the 
1 SRP also plays an important role on the s/c's orbital motion and its uncertainty should

2 be analyzed in detail, especially for s/c with large solar sails.

\subsection{New mission scenario - microprobes}

4 Recently, microprobes (e.g. CubeSats, NanoSats) and their constellations are 5 popular candidates for future asteroid missions because of their low cost [102]. With

6 the limited-area solar sails and low/micro-thrusts, the transfer from high altitude

7 orbits to low ones is resultantly slow. Therefore, the s/c might be captured by the

8 main ground-track (or mean motion) resonances during its slow crossing of the

9 resonance region [103]. This brings new challenges that are not present for a s/c with

10 large maneuver capability. In addition, the lower the resonance order ${ }^{2}$ is, the stronger

11 the motion is influenced, due to the fast accumulation of perturbation from the

12 irregular gravity field within a short period of time. For instance, the deviation of the

13 position from the nominal value in the STOs around Itokawa was found at local

14 maxima around the 2:1, 3:1 ground track resonances [9]. The dynamics in the

15 resonance region is rich in terms of resonance splitting, overlap and chaos [104]. It is

16 possible that the $\mathrm{s} / \mathrm{c}$ is temporarily or permanently captured in these regions. Very

17 limited research has been performed on this topic, which is far from being enough

18 [104]. Specifically, the probability of the $s / c$ 's capture into the resonance region is

19 highly dependent on its states and the forces (e.g. SRP, gravity, maneuvers) exerting

20 on it, all of which have uncertainties. It is required to put efforts on including these

21 uncertainties in the resonance analysis. Moreover, due to the limited maneuver and

22 operational capabilities of microprobes, the uncertainty analysis for evaluating

23 mission risks and avoiding unnecessary fuel consumption is therefore very important.

\subsection{Application of the (semi-)analytical methods}

25 Uncertainty analysis (UA) characterizes how input uncertainties affect model 26 outcomes. In addition to UA, the other demand of applying new methods is the

27 determination of the individual uncertainty's contribution to the outputs, which is

28 called the sensitivity analysis (SA). The critical parameters or initial conditions that

29 drive/dominate the system dynamics can be identified from SA [60]. For UA, the

${ }^{2}$ Given the $M: N$ resonance, the resonance order is defined as the value of $|M-N|$. 
1 distributions are chosen based on our understanding of the environment and space

2 systems. In contrast, for SA the distributions can be simply selected to fully explore 3 potential variable effects. However, the implementations of both UA and SA are

4 closely connected and complementary to each other in mission design. Since the SA

5 is essential to identify the dominant uncertainties for different small body mission

6 phases (e.g. rendezvous, orbiting, hovering, proximity and landing, etc.), the most

7 suitable mission orbits, control and navigation algorithms can be better identified

8 during the design phase by addressing SA. Nevertheless, the systematic UA and SA

9 require more efficient methods for large amount of simulations, to achieve the

10 required accuracy with the highly nonlinear dynamics of small body explorations.

11 For the methods described in Section 3.1.2, the DA and STT are based on the 12 expansion or approximation of the dynamical model (dynamics-based), while in 13 contrast the UT and PC are sample-based with no requirement on simplifying the fully 14 nonlinear dynamics. One main shortage of the dynamics-based methods is that it is 15 difficult to go to very high order approximations for a high-fidelity system, due to 16 computational complexity. The other drawback is that they are local methods that are 17 not capable of handling dynamics with large uncertainties. This however can be 18 solved by using an automatic domain splitting method that automatically splits the 19 current polynomial expansion into two polynomials whenever its truncation error 20 reaches a predefined threshold. The detailed description and application can be found 21 in Wittig et al. [105]. Moreover, they require the dynamics to be continuous and 22 differentiable. Hence, they encounter problems for perturbations with discontinuity, 23 e.g. SRP (the shadow effect) and the discrete outgassing jet. The drawback of the 24 sample-based methods is that the number of samples (e.g. UT) and the number of 25 expansion terms (e.g. PC) change linearly and exponentially with the dimension of 26 input uncertainties, respectively, and is very computationally expensive for large-

27 dimensional uncertainties of complex system. In addition, UT propagates Gaussians

28 at the second order while it still assumes that the output is Gaussian, which causes

29 truncation error. On the other hand, for PC, the determination of the polynomial 30 coefficients needs solving ODEs or least-squares regression or integration on 
tensor/sparse grids, which suffers the curse of dimensionality.

2 New challenges for their applications arise in small body explorations. Firstly, the 3 dynamics is highly nonlinear as a result of the irregular gravity field and the rotation

4 status of the small body. Secondly, compared with those of Earth, uncertainties of the

5 model parameters (gravity and physical parameters) are larger because of the limited

6 measurements available and the resultant restricted accuracy. Therefore, for

7 methods based on series expansions such as the DA, to achieve accurate

8 approximation of the propagated orbits, the expansion has to go to a higher order.

9 For sample-based methods, their computational efficiencies are sensitive to the

10 dimensionality of the dynamical system. More samples and high order expansion of

11 the inputs are required to accurately capture the input uncertainties with large values

12 and numbers, especially for the highly nonlinear dynamics.

13 5.4 Other research directions

14 For small body explorations, due to the long distance of the s/c w.r.t. Earth and the 15 short time that is allowed for landing or sampling operations, autonomy is highly 16 required. Therefore, robustness plays a significant role. However, the accurate 17 quantification of uncertainties together with the efficient and accurate handling of 18 them can make sure that the mission is designed and performed robustly.

19 For instance, as mentioned in Section 1, uncertainties of the s/c's state spread in a 20 wide range during the descent phase. This is an ideal scenario for applying and testing 21 feedback control algorithms [106], due to the relatively weak gravity attraction of the 22 small body and resultantly larger thrust/maneuver authority of the s/c. The control 23 operation allows for more accurate and robust soft-landings. However, robust 24 treatment of uncertainties will be essential to reduce the resultant control efforts.

25 Their qualitative and quantitative relations should also be the focus of future research.

\section{Conclusions}

27 In this survey, the status of the small body explorations is firstly presented. Then, the 28 dynamics of the orbital motion around the small body is described, together with the 29 approaches of modeling different forces and the possible uncertainties. Afterwards, 30 the sources of uncertainties are analyzed and are classified into the aleatory and 
1 epistemic categories, including uncertainties in $s / c$ 's state, operations, gravity and

2 outgassing of the small body, SRP, etc.. For the arrival, orbiting, hovering, proximity

3 and landing phases, the main uncertainties for each phase are discussed. The MC,

4 linear method and nonlinear methods of uncertainty characterizations are described.

5 The nonlinear methods are analyzed to have great potential and advantages of

6 application in small body missions. Future research efforts, e.g. complete modeling of

7 uncertainties and applying new and more efficient methods and microprobes, are put

8 forward for reference.

\section{Acknowledgement}

11 This work is funded by the Special Financial Grant of Chinese Postdoc Foundation and 12 Natural Science Foundation of China for Young Scientist with No. 11703013. X. Hou 13 wishes to thank the support from the Natural Science Foundation of China with No. 1411773017.

\section{References}

17 [1] D. Scheeres, Orbital Motion in Strongly Perturbed Environments, Springer, 2012.

18 [2] M.Belton, C. Chapman, K. Klaasen, A. Harch, P. Thomas, J. Veverka, A. Mcewen, R.

19 Pappalardo, Galileo's encounter with 243 Ida: An overview of the imaging experiment, 20 Icarus, 120 (1996) 1-19.

21 [3] H. Keller, C. Barbieri, D. Koschny, P. Lamy, H. Rickman, The Rosetta Asteroid Šteins

22 Flyby observed by OSIRIS, Bulletin of the American Astronomical Society, 40 (2008) 23442.

24 [4] Y. Zhao, J. Ji, J. Huang, S. Hu, X. Hou, Y. Li, W. Ip, Orientation and rotational 25 parameters of asteroid 4179 Toutatis: new insights from Chang'e-2's close flyby, 26 Monthly Notices of the Royal Astronomical Society, 450 (2015) 3620-3632.

27 [5] J. Veverka, M. Robinson, P. Thomas, S. Murchie, J. Bell, N. Izenberg, C. Chapman, A.

28 Harch, M. Bell, B. Carcich, NEAR at Eros: Imaging and spectral results, Science, 289 29 (2000) 2088-2097.

30 [6] A. Fujiwara, J. Kawaguchi, D. Yeomans, M. Abe, T. Mukai, T. Okada, J. Saito, H. Yano, 
1 M. Yoshikawa, D. Scheeres, The rubble-pile asteroid Itokawa as observed by 2 Hayabusa, Science, 312 (2006) 1330-1334.

3 [7] T. Kubota, T. Hashimoto, J. Kawaguchi, M. Uo, K. Shirakawa, Guidance and

4 Navigation of Hayabusa Spacecraft for Asteroid Exploration and Sample Return

5 Mission, International Joint Conference on SICE-ICASE , 45(2) (2007) 2793-2796.

6 [8] N. Croisard, M. Vasile, S. Kemble, G. Radice, Preliminary space mission design

7 under uncertainty, Acta Astronautica, 66 (2010) 654-664.

8 [9] M. Ionescu-Bujor, D. Cacuci, A Comparative Review of Sensitivity and Uncertainty

9 Analysis of Large-Scale Systems--I: Deterministic Methods, Nuclear Science and 10 Engineering. 147(3) (2004) 189-2003.

11 [10] A. Fuller, Analysis of nonlinear stochastic systems by means of the Fokker12 Planck equation, Int. J. Control, 9 (6) (1969) 603-655.

13 [11] D. Cacuci, M. Ionescu-Bujor, A Comparative Review of Sensitivity and Uncertainty

14 Analysis of Large-Scale Systems--II: Statistical Methods, Nuclear Science and 15 Engineering, 147(3) (2004) 204-217.

16 [12] Y. Luo, Z. Yang, A review of uncertainty propagation in orbital mechanics,

17 Progress in Aerospace Sciences, 89 (2017) 23-29.

18 [13] A. Harris, M. Gargaud, W. Irvine, et al., Asteroid, Encyclopedia of Astrobiology, 19 Springer Berlin Heidelberg, 2015.

20 [14] M. Belton, N. Samarasinha, Y. Fernndez, K. Meech, The excited spin state of Comet 21 2P/Encke," Icarus, 175(2005) 181-193.

22 [15] D. Scheeres, F. Marzani, A. Rossi, Evolution of NE0 rotation rates due to close 23 encounters with Earth and Venus, Icarus, 170(2004) 312-323.

24 [16] P. Pravec, A. Harris, P. Scheirich, P. Kusnireka, L. Sarounov, C. Hergenrother, S. 25 Mottola, M. Hickse, G. Masi, Y. Krugly, V. Shevchenko, M. Nolani, E. Howell, M. 26 Kaasalainen, A. Galed, P. Brown, D. DeGraff, J. Lambert, S. Foglia, Tumbling asteroids, 27 Icarus, 173(2005) 108-131.

28 [17] S. Ostro, R. Hudson, R. Rosema, K.Giogini, J. Jurgens, R. Yeomans, D. Chodas, P. 29 Winkler, R. Rose, R. Choate, Asteroid 4179 Toutatis: 1996 radar observations, Icarus, 30 137(1999) 122-139. 
1 [18] Y. Zhao, J. Ji, J. Huan, S. Hu, X. Hou, Y. Li, W. IP, Orientation and rotational

2 parameters of asteroid 4179 Toutatis: new insights from Chang'e-2's close flyby,

3 Monthly Notices of the Royal Astronomical Society, 450(2015) 3620-3632.

4 [19] S. Byram. The Effects of Outgassing Jets on the Rotation of a Comet Nucleus and

5 on the Trajectory of an Orbiting Spacecraft. PhD thesis, The University of Michigan,

62008.

7 [20] W. Kaula, Theory of satellite geodesy: applications of satellites to geodesy,

8 Massachusetts, Blaisdell Publishing Company, 1966.

9 [21] W. Macmillan, The theory of the potential, New York, Dover, 1958.

10 [22] R. Werner, The gravitational potential of a homogeneous polyhedron or don't cut

11 corners. Celestial Mechanics and Dynamical Astronomy, 59(1994) 253-278.

12 [23] R. Werner, D. Scheeres, Exterior gravitation of a polyhedron derived and

13 compared with harmonic and mascon gravitation representations of asteroid 4769

14 Castalia. Celestial Mechanics and Dynamical Astronomy, 65(1997) 313-344.

15 [24] R. Park, R. Werner, S. Bhaskaran, Estimating small-body gravity field from shape

16 model, and navigation data. Journal of Guidance, Control, and Dynamics, 33(2010),

$17 \quad 212-221$.

18 [25] P. Muller, W. Sjogren, Mascons: Lunar mass concentrations. Science, 161(1968)

19 680-684.

20 [26] M. Maruya, H. Ohyama, M. UO, N. Muranaka, H. Morita, T. Kubota, T. Hashimoto,

21 J. Saito, J. Kawaguchi, Navigation shape and surface topography model of Itokawa.

22 AIAA Guidance, Navigation, and Control Conference, 2006.

23 [27] W. Press, Numerical recipes 3rd edition: The art of scientific computing,

24 Cambridge University Press, 2007.

25 [28] A. Riaguas, A. Elipe, M. Lara, Periodic Orbits around a Massive Straight Segment.

26 Celestial Mechanics and Dynamical Astronomy, 73(1999) 169-178.

27 [29] P. Bartczak, P. Breiter, Double material segment as the model of irregular bodies.

28 Celestial Mechanics and Dynamical Astronomy, 86(2003) 131-141.

29 [30] M. Hirabayashi, M. Morimoto, H. Yano, J. Kawaguchi, Linear stability of collinear

30 equilibrium points around an asteroid as a two-connected-mass: Application to fast 
1 rotating Asteroid 2000EB 14, Icarus, 206(2010) 780-782.

2 [31] A. Milani, A. Nobill, P. Farinella, Non-gravitational perturbations and satellite 3 geodesy. Adam Hilger Ltd.,Accord, MA, 1987.

4 [32] C. McInnes, Solar Sailing: Technology, Dynamics and Mission Applications, 5 Springer, 2004.

6 [33] M. Ziebart, High precision analytical solar radiation pressure modelling for GNSS 7 spacecraft. PhD thesis, University of East London, 2001.

8 [34] M. Ziebart and P. Dare, Analytical Solar Radiation Pressure Modelling for 9 GLONASS using a pixel array, Journal of Geodesy, 75(2001) 587-599.

10 [35] S. Hesar, D. Scheeres, J. McMahon, Precise Solar Radiation Pressure Models for

11 Small-Body Orbiters: Applications to OSIRIS-REx Spacecraft, Journal of Guidance, 12 Control, and Dynamics, 40(7) (2017) 1638-1650.

13 [36] J. McMahon, D. Scheeres, New Solar Radiation Pressure Force Model for 14 Navigation, Journal of Guidance, Control and Dynamics, 33(2010) 1418-1429.

15 [37] A. Farrés, D. Folta, C. Webster, Using spherical harmonics to model solar 16 radiation pressure accelerations, Advances in the Astronautical Sciences, 162 (2018) $17 \quad 3365-3383$.

18 [38] D. Scheeres, F. Marzari, L. Tomasella, V. Vanzani. ROSETTA mission: satellite 19 orbits around a cometary nucleus. Planetary and Space Science, 46(1998) 649-671.

20 [39] S. Byram. The Effects of Outgassing Jets on the Rotation of a Comet Nucleus and 21 on the Trajectory of an Orbiting Spacecraft. PhD thesis, The University of Michigan, 222008.

23 [40] A. Neishtadt, D. Scheeres, V. Sidorenko, P. Stooke, A. Vasiliev, Evolution of Comet 24 Nucleus Rotation, Icarus, 157(2002) 205-218.

25 [41] B. Marsden, Z. Sekanina, D. Yeomans, Comets and non-gravitational forces, The 26 Astronomical Journal, 78(1973) 211-225.

27 [42] G. Beekman,. I.O. Yarkovsky and the discovery of 'his' effect. Journal for the 28 History of Astronomy, 37(2006) 71-86.

29 [43] D. Rubincam, Radiative spin-up and spin-down of small asteroids. Icarus, $30 \quad 148(2000) 2-11$. 
1 [44] W. Bottke, D. Vokrouhlicky, D. Rubincam, et al., The Yarkovsky and Yorp effects:

2 Implications for asteroid dynamics. Annual Review of Earth and Planet Sciences, 3 34(2006) 157-191.

4 [45] D. Vokrouhlicky, W. Bottke, S. Chesley, et al., The Yarkovsky and YORP Effects.

5 Asteroids IV, University of Arizona Press, Tucson, (2015) 509-531.

6 [46] D. Rubincam, LAGEOS orbit decay due to infrared radiation from Earth. Journal

7 of Geophysical Research, 92(1987) 1287-94.

8 [47] D. Scheeres, S. Ostro, R. Hudson, R. Werner, Orbits close to asteroid 4769 Castalia.

9 Icarus, 121(1996) 67-87.

10 [48] D. Scheeres, S. Ostro, R. Hudson, E. Dejong, S. Suzuki, Dynamics of orbits close to 11 asteroid 4179 Toutatis. Icarus, 132(1998) 53-79.

12 [49] J. Feng, R. Noomen, J. Yuan, Orbital Motion in the Vicinity of the Non-collinear 13 Equilibrium Points of a Contact Binary Asteroid. Planetary and Space Science, 14 117(2015a) 1-14.

15 [50] J. Feng, X. Hou. Dynamics around equilibrium points of uniformly rotating 16 asteroids. The Astronomical Journal, 154(1) (2017): 21.

17 [51] J. Feng, R. Noomen, P. Visser, J. Yuan, Modeling and analysis of periodic orbits 18 around a contact binary asteroid. Astrophysics and Space Science, 357(2015b) 1-18.

19 [52] Y. Yu, H. Baoyin, Generating families of 3D periodic orbits about asteroids. 20 Monthly Notices of the Royal Astronomical Society, 427(2012) 872-881.

21 [53] S. Broschart, B. Villac, Identification of non-chaotic terminator orbits near 6489 22 Golevka. Advances in Astronautical Sciences, 134(2009) 861-880.

23 [54] D. Scheeres, B. Williams, W. Bollman, R. Davis, C. Helfrich, S. Synnott, D. Yeomans.

24 Navigation for low-cost missions to small solar system bodies, Acta Astronautica, 35 25 (1995) 211-220.

26 [55] J. Miller, B. Williams, W. Bollman, R. Davis, C. Helfrich, D. Scheeres, S. Synnott, T.

27 Wang, D. Yeomans, Navigation analysis for Eros rendezvous and orbital phases, 28 Journal of the Astronautical Sciences, 43(4) (1995) 453-476.

29 [56] D. Scheeres, Design and Analysis of Landing Trajectories and Low-Altitude 30 Asteroid Flyovers, IPN Progress Report, 2001, 42-146. 
1 [57] P. Maybeck, Stochastic Models, Estimation, and Control, Academic press, New 2 York, 1982.

3 [58] M. Spiegel, J. Schiller, R. Srinivasan, Probability and Statistics, third edition, 4 McGraw-Hill, 2009.

5 [59] G. Shafer, A Mathematical Theory of Evidence, Princeton University Press, 6 Princeton, 1976.

7 [60] S. Marino, I. Hogue, C. Ray, D. Kirschner, A methodology for performing global 8 uncertainty and sensitivity analysis in systems biology, Journal of Theoretical Biology, 9254 (2008) 178-196.

10 [61] W.L. Oberkampf, J.C. Helton, Investigation of evidence theory for engineering 11 applications, in: Fourth Non-Deterministic Approaches Forum, vol. 1569, AIAA, April 122002.

13 [62] M. Vasile, Robustness optimization of aerocapture trajectory design using a 14 hybrid co-evolutionary approach, in: 18th International Symposium on Space Flight 15 Dynamics, vol. 548, ESA Special Publication, 2004.

16 [63] M. Vasile, D. Bonetti, Evolution of the concurrent design process under 17 uncertainties, in: International Concurrent Engineering Workshop, ESA, 2004.

18 [64] A. Fuller, Analysis of nonlinear stochastic systems by means of the Fokker19 Planck equation, International Journal of Control, 9 (6) (1969) 603-655.

20 [65] C. Mooney, Monte Carlo simulation, SAGE Publication, 1997.

21 [66] R. Battin, An Introduction to the Mathematics and Methods of Astrodynamics, 22 AIAA (American Institute of Aeronautics and Astronautics), Education Series, 1999.

23 [67] M. Berz, Modern Map Methods in Particle Beam Physics, Academic Press, New 24 York, 1999, 82-96.

25 [68] R. Armellin, P. Di Lizia, F. Bernelli-Zazzera, M. Berz, Asteroid close encounters 26 characterization using differential algebra: the case of Apophis, Celestial Mechanics 27 and Dynamical Astronomy, 107(4) (2010) 451-470.

28 [69] A. Morselli, R. Armellin, P. Di Lizia, F. Bernelli-Zazzera, A high order method for 29 orbital conjunctions analysis: sensitivity to initial uncertainties, Advances in Space 30 Research, 53 (3) (2014) 490-508. 
1 [70] P. Di Lizia, R. Armellin, A. Ercoli-Finzi, M. Berz, High-order robust guidance of 2 interplanetary trajectories based on differential algebra, J. Aerosp. Eng. Sci. Appl.,1 3 (1) (2008) 43-57.

4 [71] P. Di Lizia, R. Armellin, F. Bernelli-Zazzera, M. Berz, High order optimal control 5 of space trajectories with uncertain boundary conditions, Acta Astronautica, 93 (2014) $6 \quad 217-229$.

7 [72] R. Park, D. Scheeres, Nonlinear mapping of Gaussian statistics: theory and 8 applications to spacecraft trajectory design, Journal of Guidance, Control and 9 Dynamics, 29(6) (2006) 1367-1375.

10 [73] I. Park, K. Fujimoto, D. Scheeres, Effect of dynamical accuracy for uncertainty 11 propagation of perturbed keplerian motion, Journal of Guidance, Control and 12 Dynamics, 38(12) (2015), 2287-2300.

13 [74] K. Fujimoto, D. Scheeres, K. Alfriend, Analytical nonlinear propagation of 14 uncertainty in the two-body problem, Journal of Guidance, Control and Dynamics, 15 35(2) (2012) 497-509.

16 [75] S. Julier, J. Uhlmann, H. Durrant-Whyte, A new approach for filtering nonlinear 17 systems, in: Proceedings of IEEE American Control Conference, 1995, 1628-1632.

18 [76] S. Julier, J. Uhlmann, Unscented filtering and nonlinear estimation, Proc. IEEE 19 92(3) (2004) 401-422.

20 [77] N. Wiener, The homogeneous chaos, Am. J. Math., 60(4) (1938) 897-936.

21 [78] B. Jones, A. Doostan, G. Born, Nonlinear propagation of orbit uncertainty using 22 non-intrusive polynomial chaos, Journal of Guidance, Control and Dynamics, 36(2) 23 (2013) 430-444.

24 [79] A. Prabhakar, J. Fisher, R. Bhattacharya, Polynomial chaos-based analysis of 25 probabilistic uncertainty in hypersonic flight dynamics, Journal of Guidance, Control 26 and Dynamics, 33(1) (2010) 222-234.

27 [80] J. Miller, A. Konopliv, P. Antreasian, J. Bordi, S. Chesley, C. Helfrich, W. Owen, T. 28 Wang, B. Williams, D. Yeomans, D. Scheeres, Determination of Shape, Gravity, and 29 Rotational State of Asteroid 433 Eros, Icarus, 155 (2002) 3-17.

30 [81] M. Rayman, T. Fraschetti, C. Raymond, C. Russell, Dawn: A mission in 
1 developments for exploration of main belt asteroids Vesta and Ceres, Acta 2 Astronautica, 58 (2006) 605-616.

3 [82] J. Kawaguchi, A. Fujiwara, T. Uesugi, Hayabusa-Its technology and science 4 accomplishment summary and Hayabusa-2, Acta Astronautica, 62 (2008) 639-647.

5 [83] J. Melman, E. Mooij, R. Noomen, State propagation in an uncertain asteroid 6 gravity field, Acta Astronautica, 91 (2013) 8-19.

7 [84] S. Watanabe et al., Hayabusa2 arrives at the carbonaceous asteroid Ryugu-A 8 spinning top-shaped rubble pile, Science 10.1126/science.aav8032, 2019.

9 [85] H. Ikeda, N. Hirata, R. Nakamura, Y. Tsuda, T. Iwata, M. Abe, M. Yoshikawa,

10 Preliminary Analysis of Small-body Gravity Estimation in the Hayabusa-2 Mission, the 11 24th JAXA workshop on astrodynamics and flight mechanics, 2014.

12 [86] S. Watanabe, Y. Tsuda, M. Yoshikawa, S. Tanaka, T. Saiki, S. Nakazawa, Hayabusa132 Mission Overview, Space Science Reviews, 208(1-4) (2017) 3-16.

14 [87] http://www.hayabusa2.jaxa.jp/en/news/status/, retrieved on 1st April, 2019.

15 [88] http://sci.esa.int/rosetta/54456-highlights-from-the-rosetta-mission-thus-far/, 16 retrieved on 1st April, 2019.

17 [89] M. Patzold, et al., A homogeneous nucleus for comet 67P/Churyumov18 Gerasimenko from its gravity field, Nature 10.1038/nature16535, 2016.

19 [90] C. Lhotka, S. Reimond, J. Souchay, et al. Gravity field and solar component of the 20 precession rate and nutation coefficients of Comet 67P/Churyumo-Gerasimenko, 21 Monthly Notices of the Royal Astronomical Society, 455(2016) 3588-3596.

22 [91] D. Scheeres, et al., The dynamic geophysical environment of (101955) Bennu

23 based on OSIRIS-Rex measurements, Nature Astronomy, 3(2019) 352-361.

24 [92] http://www.asteroidmission.org/status-updates/, retrieved on 1st April, 2019.

25 [93] S. Hesar, D. Scheeres, J. Mcmahon, Sensitivity Analysis of the OSIRIS-REx 26 Terminator Orbits to Maneuver Errors, Journal of Guidance Control Dynamics, 40 27 (2017) 1-15.

28 [94] K. Berry, B. Sutter, A. May, K. Williams, Brent Barbee, M. Beckman, B. Williams, 29 OSIRIS-REx Touch-And-Go (TAG) Mission Design and Analysis, Advances in the 30 Astronautical Sciences, 149 (2013) 667-678. 
1 [95] C. W. Hergenrother, The operational environment and rotational acceleration of 2 asteroid (101955) Bennu from OSIRIS-REx observations, Nature Communications, 3 volume 10, article number: 1291 (2019).

4 [96] A. Cheng, J. Atchison, B. Kantsiper, A. Rivkin, A. Stickle, C. Reed, A. Galvez, I.

5 Carnelli, P. Michel, S. Ulamec, Asteroid Impact and Deflection Assessment mission, 6 Acta Astronautica, 115 (2015) 262-269.

7 [97] P. Michel, M. Kueppers, A. Cheng, The Hera mission: European component of the 8 Asteroid Impact and Deflection Assessment (AIDA) mission to a binary asteroid, 49th 9 Lunar and Planetary Science Conference, No. 1144, 2018.

10 [98] M. Hirabayashi, A. Davis, S. Naidu, Y. Yu, E. Fahnestock, S. Schwartz, D. Richardson, 11 P. Michel, D. Scheeres, S. Chesley, A. Cheng, A. Rivkin, L. Benner, NASA's DART mission 12 to Didymos: the effect of shape deformation of the primary and ellipticity of the 13 secondary on post-impact orbital period, 49th Lunar and Planetary Science 14 Conference, No. 2108, 2018.

15 [99] F. Damme, H. Hussmann, J. Oberst, Spacecraft orbit lifetime within two binary 16 near-Earth asteroid systems, Planetary and Space Science, 146 (2017) 1-9.

17 [100] O. Celik, O. Karatekin, B. Ritter, J. Sanchez, Reliability Analysis of Ballistic 18 Landing in Binary Asteroid 65803 (1996GT) Didymos under Uncertainty and GNC 19 Error, Transactions of JSASS, Aerospace Technology Japan, 14(31) (2017) 1-10.

20 [101] J. Feng, X. Hou, The semi-analytical analysis of orbital evolution around an 21 asteroid under the effects of the C20 term, the solar radiation pressure and the 22 asteroid's orbital eccentricity, Advances in Space Research, 62(9)(2018) 2649-2664.

23 [102] A. Poghosyan, A. Golkar, CubeSat evolution: Analyzing CubeSat capabilities for 24 conducting science missions, Progress in Aerospace Sciences, 88 (2017) 59-83.

25 [103] N. Delsate, Analytical and numerical study of the ground-track resonances of 26 Dawn orbiting Vesta. Planetary and Space Science, 59 (2011) 1372-1383.

27 [104] A. Morbidelli, Modern celestial mechanics: aspects of solar system dynamics, 28 London: Taylor \& Francis, 2002.

29 [105] A. Wittig, P. Di Lizia, R. Armellin, et al., Propagation of large uncertainty sets in 30 orbital dynamics by automatic domain splitting, Celestial Mechanics and Dynamical 
1 Astronomy, 122(3)(2015) 239-261.

2 [106] W. Dunham, C. Petersen, I. Kolmanovsky, Constrained Control for Soft Landing

3 on an Asteroid with Gravity Model Uncertainty, American Control Conference, 2016,

$4 \quad 5842-5847$.

5 


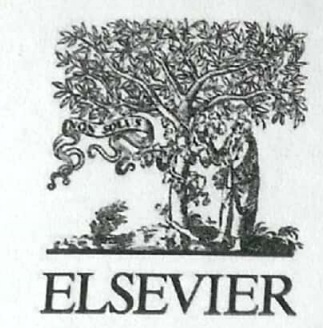

Dr. Jinglang Feng

Nanjing University

Nanjing, China

Email: iinglang@niu.edu.cn

Dear Dr. Jinglang Feng,

\section{Re: Progress in Aerospace Sciences}

Title: Survey on studies about model uncertainties in small body explorations

Type: Full Length Review

Estimated submission date: 31 December 2018

1. We are very pleased that you have agreed with Professor Max Platzer to write the above-mentioned contribution.

2. Elsevier Ltd undertakes to publish at its own risk and expense the above contribution for world-wide distribution and sales through its international marketing organization.

3. It is further understood that you will submit your contribution in the English language and in duplicate to the Commissioning Editor for refereeing, together with all diagrams and tables. For assistance with the English Language, please see the Elsevier Author Gateway Language Editing section (http://authors.elsevier.com/LanguageEditing.html).

4. Elsevier, Inc. encourages the use of its online submission and tracking system called EVISE, which allows the author to upload files directly from their computer. We strongly encourage all authors to use EVISE at the following URL when submitting papers to the journal. EVISE can be accessed at http://www.evise.com/evise/faces/pages/navigation/NavController.jspx?JRNL ACR=JPAS

(First time users will need to register).

5. In order to avoid delays in publication, please correct and return the proofs promptly to the Production Editor, Elsevier Ltd, alterations must be kept to an absolute minimum.

6. You will receive 25 free offprints of your contribution and also be entitled to order copies of any book published by Elsevier Ltd at the Author's privileged discount of $30 \%$ provided the order is placed with the Publisher specifically claiming this discount.

7. On publication of the contribution, you will receive an extra 10 complimentary copies of the issue containing your paper courtesy of Progress in Aerospace Sciences and Elsevier Ltd. 
It is understood that you will assign the copyright of the article to the Publisher in agreement of this contract.

8. You are eligible to have you figures published FREE OF CHARGE online if electronic colour files are provided to Production in keeping with the Artwork guidelines provided by the Publisher. Free online colour figures will be printed in black and white in the hardcopy journal unless colour figures in PRINT are requested.

9. You should check with your own organisation whether there are any regulations or contractual provisions governing publication of your work and should ensure that the appropriate clearance is obtained. Any special requirements concerning copyright or remuneration should be communicated to Elsevier Ltd when returning this agreement.

10. The copyright in your contribution will be protected by registering it in the name of Elsevier Ltd together with the other contributions published in the journal.

11. If you use any copyrighted material (text or illustration) in your article, you agree to supply with your script and, at your own expense, written permission from the copyright holder for its use.

If you agree to the terms of this letter I should be grateful if you would confirm acceptance by signing both copies, keeping one for your files and returning one to Yingyi Liu, Elsevier, Unit 1-6, $7^{\text {th }}$ Floor W1 Oriental Plaza, No. 1 East Chang An Ave., Dong Cheng District, Beijing 100738 China. Tel.:+861085208834,E-mail: y.liu.4@elsevier.com.

Yours sincerely

For and on behalf of Elsevier Ltd
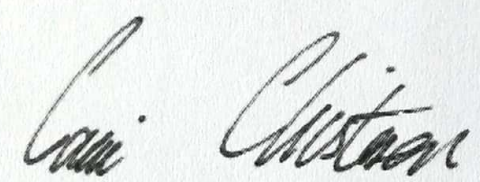

October $30^{\text {th }} 2018$

Carrie Christensen

Date

Publisher

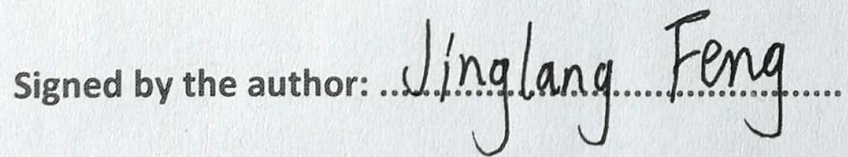

Date.... October....31 $31^{\text {th }} 2018$ 